\title{
Primera aproximación al conocimiento de las comunidades coralinas de los complejos arrecifales de Serranilla, Bajo Alicia y Bajo Nuevo-Colombia, sección norte de la Reserva de Biósfera Seaflower, Caribe Occidental
}

\section{First approach to the knowledge of Serranilla, Alice Shoal and New Shoal coral reef complex, Colombia, northern section of the Seaflower Biosphere Reserve, Western Caribbean}

\author{
Alfredo Abril-Howard ${ }^{1 *}$, Carlos Orozco Toro ${ }^{2}$, Nacor Bolaños Cubillos ${ }^{1}$ y Heins Bent Hooker ${ }^{1}$
}

\begin{abstract}
RESUMEN
Dada la dificultad de visitar dichas zonas remotas existe poca información sobre sus ecosistemas y los datos existentes provienen de desembarques de pesca industrial. Buscando establecer una aproximación al estudio de sus sistemas arrecifales, se visitaron Serranilla, Bajo Alicia y Bajo Nuevo, estimando la cobertura en 26, 14 y 42 estaciones, respectivamente. Para la caracterización se aplicó la metodología ERA, monitoreando $400 \mathrm{~m}^{2}$ por estación. La cobertura coralina de Bajo Nuevo fue de $30.2 \% \pm 19.1 \%$, y se observa un desarrollo del arrecife periférico similar al descrito para estas formaciones oceánicas en el Archipiélago. La laguna arrecifal tiene profundidades entre 6-20 m, donde el coral vivo alcanzó hasta el 70\%. En Serranilla, la cobertura coralina fue baja $(13.0 \% \pm 6.9 \%)$; la mayoría de estaciones estuvieron dominadas por macroalgas $(43.8 \% \pm 15.7 \%)$ y existe una alta dominancia del género Gorgonia $(20.8 \% \pm 15.2 \%)$. La mayor cobertura coralina se encontró en la fracción más extensa de la barrera (NW). En Bajo Alicia, el arrecife no emerge y es dominado por macroalgas $(32.9 \% \pm 17.1 \%)$; el desarrollo coralino $(15.7 \% \pm 7.9 \%)$ está asociado a grietas o elevaciones de la matriz calcárea. Actualmente, las condiciones de los arrecifes del Archipiélago son similares, aunque el aislamiento juega un papel importante en la conservación de Bajo Nuevo, donde se encuentra la cobertura de coral duro más alta de la RB Seaflower.
\end{abstract}

Palabras claves: Comunidad coralina, Reserva de Biósfera Seaflower, Archipiélago de San Andrés, Providencia y Santa Catalina, Banco Serranilla, Bajo Alicia y Bajo Nuevo.

\begin{abstract}
Given the difficulty to visit Serranilla, Alice Shoal, and New Shoal, information about their ecosystems is limited and the only data available comes from landings of the industrial fishing fleet. We visited these areas in order to study their reef systems; coral cover was estimated at 26,14, and 42 stations, respectively. Characterization was made using the ERA method, monitoring $400 \mathrm{~m}^{2}$ per station. Coral cover in New Shoal was $30.2 \% \pm 19.1 \%$, and peripheral reefs showed similar growth to the ones described for other similar oceanic formations in the Archipelago. The lagoon has depths between 6-20 m, where living coral reached up to $70 \%$. In Serranilla, coral cover was low $(13.0 \pm 6.9 \%)$, macroalgae was dominant in most of the stations $(43.8 \% \pm 15.7 \%)$, and the genus Gorgonia was highly dominant $(20.8 \pm 15.2 \%)$. The highest coral cover was found in the largest fraction of the barrier (NW). The Alice Shoal reef is permanently submerged and

1 Corporación para el Desarrollo Sostenible del Archipiélago de San Andrés, Providencia y Santa Catalina (CORALINA). Km 26 vía San Luis San Andrés, Isla Colombia. alfredoabril@hotmail.com*, nacorwbc@yahoo.com, benthoo@hotmail.com

2 Universidad Nacional de Colombia sede Caribe, Vía Circunvalar San Luis, San Andrés Isla Colombia. calotoro@yahoo.com
\end{abstract}

Recibido 30-IV-2011

Aceptado 1-VIII-2012

DOI: http://dx.doi.org/10.15359/revmar.4.3 
is dominantly covered by macroalgae $(32.9 \% \pm 17.1 \%)$; coral growth $(15.7 \% \pm 7.9 \%)$ is associated with cracks and elevations of the calcareous matrix. Currently, reef conditions in the Archipelago are similar, although isolation plays a central role in the conservation of New Shoal, where the hard coral cover is the largest in the entire Seaflower Biosphere Reserve.

Keywords: Coral community, Seaflower Biosphere Reserve, Archipelago of San Andrés, Providence and Santa Catalina, Serranilla Bank, Alice Shoal and New Shoal

\section{INTRODUCCIÓN}

El Archipiélago de San Andrés, Providencia y Santa Catalina forma parte del territorio insular colombiano, localizado aproximadamente entre $\operatorname{los} 12^{\circ}$ y $16^{\circ}$ de latitud Norte $(\mathrm{N})$ y $\operatorname{los} 78^{\circ}$ y $82^{\circ}$ de longitud Oeste (W). Comprende tres pequeñas islas habitadas (San Andrés, Providencia y Santa Catalina), seis bancos (Albuquerque, Bolívar o Courtown, Quitasueño, Serrana, Roncador y Serranilla) y dos bajos (Bajo Alicia y Bajo Nuevo) (Mow et al. 2002). Debido a la ubicación del Archipiélago, el mar territorial de Colombia en el Caribe tiene un área aproximada de $532162 \mathrm{~km}^{2}$, de los cuales $180000 \mathrm{~km}^{2}$ forman parte del Departamento (SIGCORALINA, 2010); esta área representa cerca del $34 \%$ y allí se encuentra más del $77 \%$ de la extensión total de las áreas coralinas de Colombia (INVEMAR, 2005).

Según Díaz (2005), dichos bancos y bajos son el resultado de la actividad volcánica durante el cenozoico temprano, la acumulación de caliza arrecifal sobre las cimas someras y la subsidencia de basamento entre el cenozoico y cuaternario. Se extienden sobre la elevación de Nicaragua con una longitud aproximada de 500 $\mathrm{km}$, donde desarrollan dos barreras que rodean las islas principales, cinco grandes atolones y otros bancos coralinos menos definidos (Geister y Díaz, 1997). En general, los complejos arrecifales fueron formados por los mismos procesos geológicos y aunque existen ciertos rasgos comunes en la zonación de los arrecifes coralinos, cada bajo, banco o isla mayor tiene diferencias morfológicas (Díaz et al. 1996a; 2000). El arrecife periférico o de barrera desarrollada en el costado de barlovento, terraza prearrecifal amplia se extiende hasta $20 \mathrm{~m}$ de profundidad, seguido por un talud con una pendiente marcada hasta 35-40 $\mathrm{m}$ de profundidad por una terraza y posteriormente continúa a profundidades abisales. La terraza lagunar se extiende a sotavento del arrecife periférico o de barrera, es una amplia llanura en la cual se acumulan escombros y sedimentos provenientes de la parte frontal del arrecife, seguido de esto existe una depresión topográfica con profundidades hasta de $20 \mathrm{~m}$ de profundidad, esta zona es denominada la cuenca lagunar y en ella se desarrollan parches coralinos que pueden llegar hasta la superficie. En el oeste, las lagunas son por lo general abiertas y posteriormente a la cuenca lagunar se encuentra la terraza prearrecifal y el talud de sotavento, pocas veces se presentan elevaciones de rompiente, las cuales son llamadas segmentos del arrecife periférico de sotavento (Díaz, 2005).

Serranilla, Bajo Alicia y Bajo Nuevo son tres bancos arrecifales ubicados en la zona norte del Archipiélago, se encuentran como un enclave en la zona de régimen común con Jamaica, son altamente explotados por los pescadores de Pedro Bank, debido a su cercanía, aunque es desconocido el impacto de la pesca sobre el ecosistema arrecifal. 
Teniendo en cuenta la complejidad de los ecosistemas presentes, sumado a la importancia de algunos aspectos socioculturales, el Archipiélago fue declarado por la UNESCO en el 2000 como la Reserva de la Biósfera (RB) Seaflower, tomando los mismos límites de su jurisdicción (Fig. 1). Debido a que presenta una de las más altas diversidades y endemismo en el Caribe (Acero-P. y Garzón-Ferreira, 1994), cinco años después del establecimiento de la RB Seaflower, el Ministerio de Ambiente, Vivienda y Desarrollo
Territorial creó el Área Marina Protegida Seaflower en su interior con una extensión de $65000 \mathrm{~km}^{2}$ (SIG-CORALINA, 2010), por lo cual se cataloga entre las 10 más grandes del mundo.

Además de tener una localización estratégica y privilegiada desde el punto de vista ecológico, las AMP Seaflower tienen barreras coralinas y arrecifes periféricos que juntos se extienden por aproximadamente $223 \mathrm{~km}$ longitudinales (SIG-CORALINA, 2010), ubicados a barlovento de los complejos coralinos y formando

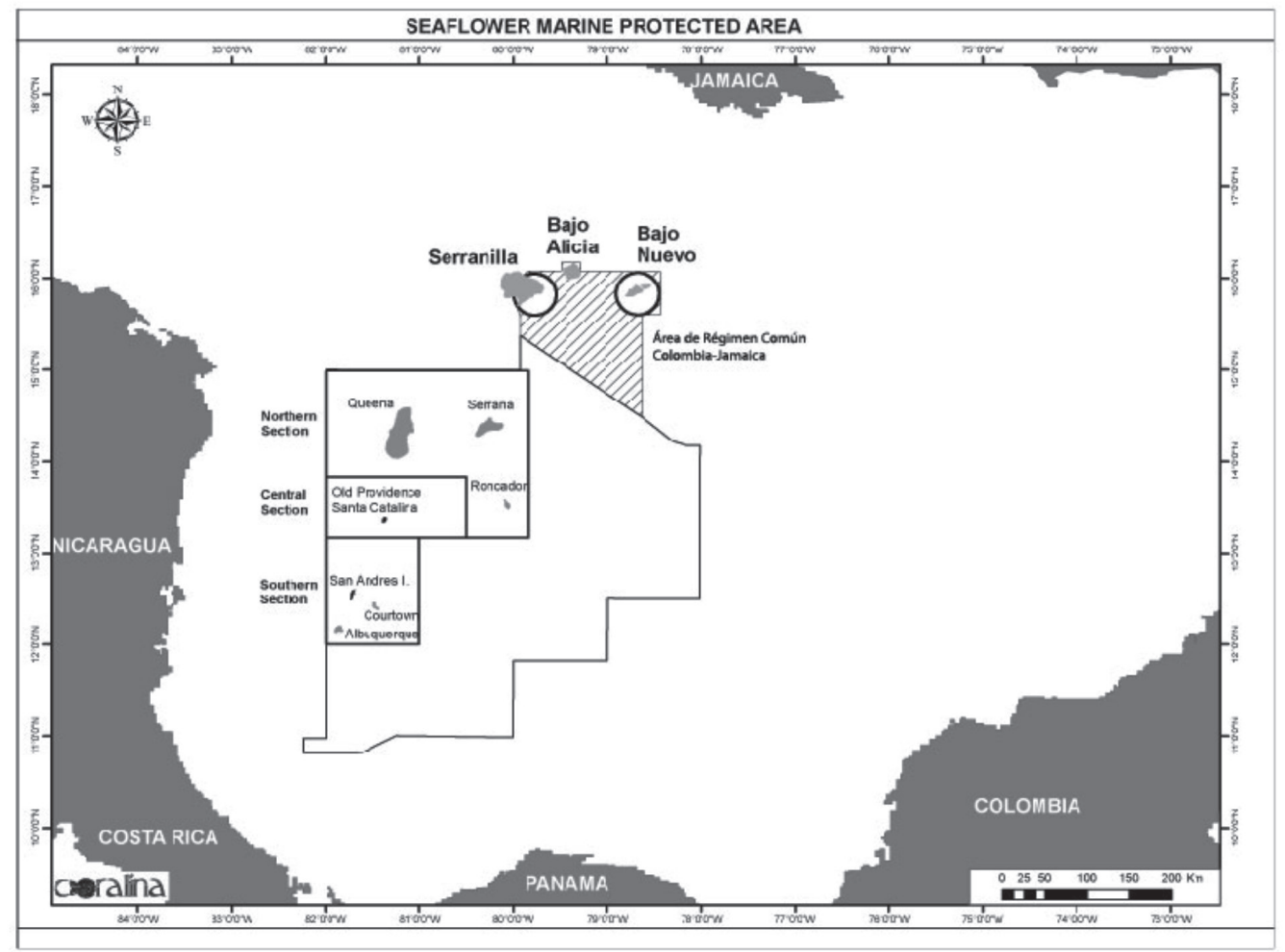

Fig. 1. Ubicación de Serranilla, Bajo Alicia y Bajo Nuevo, en la Reserva de Biósfera Seaflower (área delimitada por la línea continua), Archipiélago de San Andrés, Providencia y Santa Catalina-Colombia, el área tramada pertenece al régimen común entre Colombia y Jamaica (fuente: SIG de CORALINA, 2010)

Fig. 1. Location of Serranilla, Alice Shoal and New Shoal in the Seaflower Biosphere Reserve (area enclosed by the solid line), Archipelago of San Andrés, Providence and Santa Catalina, Colombia. The marked area belongs to the common regime between Colombia and Jamaica (source: GIS CORALINA, 2010) 
ambientes lagunares ricos en parches coralinos, ambientes protegidos profundos $\mathrm{y}$ ambientes expuestos más someros; en las islas habitadas se crean además las condiciones de sedimentación adecuadas para el desarrollo de otros ecosistemas claves como las praderas de fanerógamas marinas y bosques de manglar (Herrón, 2004).

En el Archipiélago se conocen varios trabajos sobre la comunidad coralina, como los de Barriga et al. (1969); Geister (1973); Díaz et al. (1995); Díaz et al. (1996a, b); Geister y Díaz (1997); Sánchez et al. (1997); Zea et al. (1998); Díaz et al. (2000); Chiquillo et al. (2001); Geister (2001); García y Pizarro (2002a, b); Pizarro (2002a, b, c); Dahlgren et al. (2003); Garzón-Ferreira et al. (2004); Herrón (2004); Prada (2005); Sánchez et al. (2005); Rodríguez-Ramírez et al. (2005) y Bolaños (2006).

Pese a lo anterior, los estudios puntuales de cobertura coralina, en algunas zonas remotas de la RB Seaflower como Serranilla, Bajo Alicia y Bajo Nuevo, han sido escasos, debido a las grandes distancias que tienen de las islas mayores. La mayor parte de la información existente se refiere a los desembarcos pesqueros y poco se sabe sobre el estado de los ecosistemas, siendo de vital importancia una primera aproximación a su conocimiento. En este trabajo se presenta la información de cobertura coralina recolectada durante la expedición de la zona norte de la Reserva de la Biósfera Seaflower 2010, auspiciada por la Corporación para el Desarrollo Sostenible del Archipiélago de San Andrés, Providencia y Santa Catalina (CORALINA) y la Gobernación Departamental del Archipiélago de San Andrés, Providencia y Santa Catalina.

\section{METODOLOGÍA}

\section{Área de estudio}

Serranilla (Banco Serranilla o Serranilla Bank): se localiza aproximadamente a $325 \mathrm{~km}$ al NE de Providencia y a $422 \mathrm{~km}$ de San Andrés, entre los $15^{\circ}$ $50^{\prime}$ y $16^{\circ} 04^{\prime} \mathrm{N}$ y $\operatorname{los} 80^{\circ} 03^{\prime}$ y $79^{\circ} 40^{\prime}$ W (SIG-CORALINA, 2010). Es un antiguo atolón que mide cerca de $40 \mathrm{~km}$ en longitud, $32 \mathrm{~km}$ en amplitud y un área de $1200 \mathrm{~km}^{2}$. Está compuesto por una plataforma carbonatada de unos ocho metros de profundidad, en la que sobresale hasta la superficie un arrecife periférico (tipo barrera arrecifal) fragmentado, ubicado en barlovento al SE, permitiendo en su parte trasera (sotavento) la conformación de una terraza lagunar y una laguna arrecifal. Sobre esta parte somera del Banco emergen pequeños islotes, entre los que están: West Breaker, Cayo Medio (Middle Cay), Cayo Este (East Cay) y Cayo Beacon (Beacon Cay) (Bruckner, 2012), de los cuales el primero y el cuarto tienen poca vegetación y los otros dos solo arena; Cayo Beacon es el de mayor tamaño y en él se encuentra un faro de concreto de aproximadamente $33 \mathrm{~m}$ de alto (Bruckner, 2012), que representa un elemento clave para la navegación de embarcaciones de mediano y mayor calado que transitan desde y hacia Centroamérica. La fragmentación del arrecife periférico genera condiciones ambientales variadas al interior de la laguna arrecifal del Banco, como fuertes corrientes y turbulencia.

Bajo Alicia (Banco Alicia o Alice Shoal): se localiza aproximadamente a $382 \mathrm{~km}$ al NE de Providencia, a $58 \mathrm{~km}$ de Serranilla y a $85 \mathrm{~km}$ de Bajo Nuevo, entre $\operatorname{los} 15^{\circ} 57^{\prime}$ y $16^{\circ} 10^{\prime} \mathrm{N}$ y $\operatorname{los} 79^{\circ} 28^{\prime}$ y $79^{\circ}$ 16' W (SIG-CORALINA, 2010). Es un banco coralino relativamente pequeño con 
cerca de $16 \mathrm{~km}$ de diámetro (definido por la isobata de $200 \mathrm{~m}$ ), lo que corresponde a un área de más de $200 \mathrm{~km}^{2}$. Ya que se encuentra sumergido en su totalidad, en este Bajo no hay arrecife periférico superficial ni cayos o islas emergidas. La profundidad mínima es de $11 \mathrm{~m}$, en su lado este, y en general la parte superior tiene profundidades menores a $36 \mathrm{~m}$ (Abril-Howard et al. 2010).

Bajo Nuevo (New Shoal): se localiza aproximadamente a $405 \mathrm{~km}$ al NE de Providencia, a $123 \mathrm{~km}$ de Serranilla y a 496 $\mathrm{km}$ de San Andrés, entre los $15^{\circ} 47^{\prime}$ y $15^{\circ}$ $56^{\prime} \mathrm{N}$ y los $78^{\circ} 49^{\prime}$ y $78^{\circ} 31^{\prime} \mathrm{W}$ (SIG-CORALINA, 2010). Es un complejo coralino con forma de atolón, con un arrecife periférico de cerca de $27 \mathrm{~km}$ de largo (SIGCORALINA, 2010), el cual está dividido en dos secciones que son atravesadas por un canal profundo $(60 \mathrm{~m})$ de alrededor de $1.4 \mathrm{~km}$ de ancho en su punto más estrecho. El complejo coralino más grande al SW mide $15.4 \mathrm{~km}$ de NE a SW y aproximadamente $9.4 \mathrm{~km}$ de ancho, con un área aproximada de $100 \mathrm{~km}^{2}$. El complejo arrecifal más pequeño (NE) mide $10.5 \mathrm{~km}$ de $\mathrm{E}$ a $\mathrm{W}$ y $5.5 \mathrm{~km}$ de ancho, con un área aproximada de $45 \mathrm{~km}^{2}$ (Bruckner, 2012).

\section{Evaluación de la comunidad coralina}

La expedición se llevó a cabo a bordo de la motonave "CAPITAN S" de bandera colombiana entre el 12 y el 21 de abril de 2010. Se muestrearon 82 estaciones, que fueron visitadas en embarcaciones menores, los censos se realizaron por medio de equipo autónomo SCUBA, entre 2 y $30 \mathrm{~m}$ de profundidad.

Para la caracterización de las comunidades bentónicas se utilizó la metodología de Evaluaciones Rápidas de ArrecifesERA, la cual consiste en el registro visual de la cobertura del sustrato, teniendo en cuenta categorías de fácil identificación como: arena, cascajo, corales duros, corales blandos, esponjas y macroalgas, en una parcela de $400 \mathrm{~m}^{2}(20 \times 20 \mathrm{~m})$. Adicionalmente, se registraron el número de especies (riqueza) de coral y sus enfermedades solo en los sitios donde estaban presentes en la mayoría de las especies de coral; la identificación de los corales se realizó hasta el nivel taxonómico más bajo y como medio de verificación se utilizaron las guías fotográficas de Phral y Erhardt, 1985; Humann y Deloach (2002a, b); Weil y Ruiz (2003).

\section{RESULTADOS}

\section{Serranilla}

El porcentaje de cobertura coralina en Serranilla fue el más bajo $(13.0 \%$ $\pm 6.9 \%$ ) de los tres sitios visitados. La mayoría de las estaciones estuvieron dominadas por la categoría macroalgas con $43.8 \% \pm 15.7 \%$ (Fig. 2). Aunque existió una alta cobertura de macroalgas en las zonas cercanas al arrecife periférico, también se evidenció una gran presencia de corales blandos dominados por Gorgonia spp. $(20.8 \% \pm 15.2 \%)$. En la terraza prearrecifal se destacó la dominancia de especies coralinas de crecimiento rápido, tales como Porites astreoides y Millepora alcicornis. La asociación coralina presente en la zona de rompiente del arrecife periférico fue la del zoantídeo Palythoa caribbaeorum, el hidrocoral Millepora spp. y el alga coralina incrustante Porolithon pachidemum (Palythoa-Millepora-Porolithon). En las zonas menos expuestas al oleaje, se encontró el coral Acropora palmata y costras de los corales $P$. astreoides, Siderastrea spp. y Diploria spp. En todo el arrecife periférico se observó un alto desarrollo de corales blandos. La mayor 
cobertura de especies coralinas se encontró hacia el NW, donde existe la fracción más extensa del arrecife periférico. La cobertura de algas estuvo dominada por Stypopodium zonale, Dictyota spp., Padina spp., Sargassum spp., Turbinaria turbinata y Halimeda spp.

En la laguna arrecifal no se encontró un buen desarrollo de arrecifes de parche; en su mayoría estuvo dominada por sedimentos y cascajo cubiertos por macroalgas frondosas y calcáreas (Udotea, Rhipocephalus, Halimeda, entre otras), y colonias de coral de tamaños no superiores a $\operatorname{los} 10 \mathrm{~cm}$. Hacia la zona más occidental del Banco existe una elevación de roca calcárea (segmentos del arrecife periférico de sotavento). En Serranilla se observaron 36 especies de corales formando pequeñas "cabezas" (colonias) o superficies costrosas
(Cuadro 1). El fraccionamiento de la barrera no genera condiciones de resguardo óptimas para el asentamiento de larvas en la zona de la laguna y estas condiciones de corriente y turbulencia no favorecen el desarrollo de colonias de un gran número de especies (Díaz et al. 1996a; García y Pizarro, 2002a). Pese a esto, la zonificación de los arrecifes coralinos sigue lo expuesto por Díaz et al. (1996a; 2000) para otras áreas similares, y a lo largo del Banco Serranilla se pueden observar diferentes ambientes típicos del Archipiélago (Figs. 3-4).

\section{Bajo Alicia}

Dada su profundidad, Bajo Alicia presenta colonias de coral dispersas sobre una terraza calcárea. Está dominado por una alta cobertura de algas $(32.9 \pm 17.1 \%)$ (Fig. 2) que cubren la terraza calcárea,

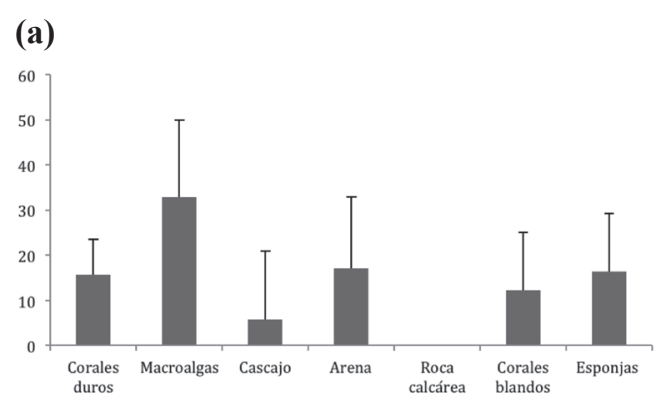

(b)

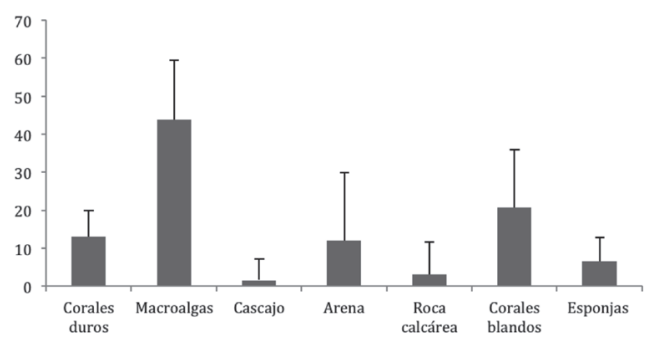

(c)

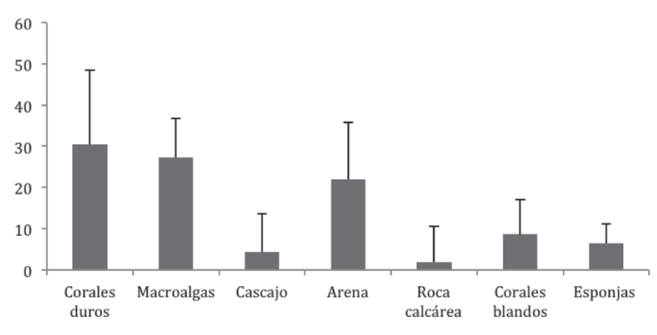

Fig. 2. Promedio de la cobertura de diferentes categorías en Bajo Alicia (a), Banco Serranilla (b) y Bajo Nuevo (c) durante el 2010

Fig. 2. Average coverage of different categories measured in the Alice Shoal (a), Serranilla Bank (b) and New Shoal (c) during 2010 
Cuadro 1. Riqueza coralina encontrada en el Banco Serranilla, Bajo Alicia y Bajo Nuevo durante el 2010

Table 1. Coral richness found in the Serranilla Bank, Alice Shoal and New Shoal during 2010

\begin{tabular}{|c|c|c|c|}
\hline Especies & Bajo Alicia & Bajo Nuevo & Serranilla \\
\hline Acropora cervicornis & & $\mathrm{x}$ & $\mathrm{x}$ \\
\hline Acropora palmata & & $\mathrm{x}$ & $\mathrm{x}$ \\
\hline Agaricia agaricites & $\mathrm{x}$ & $\mathrm{x}$ & $\mathrm{x}$ \\
\hline Agaricia fragilis & & $\mathrm{x}$ & $\mathrm{x}$ \\
\hline Agaricia lamarcki & & $\mathrm{x}$ & \\
\hline Agaricia tenuifolia & & & $\mathrm{x}$ \\
\hline Colpophyllia natans & $\mathrm{x}$ & $\mathrm{x}$ & $\mathrm{x}$ \\
\hline Dendrogira cilindrus & $\mathrm{x}$ & $\mathrm{x}$ & $\mathrm{x}$ \\
\hline Dichocoenia stokesii & $\mathrm{x}$ & $\mathrm{x}$ & $\mathrm{x}$ \\
\hline Diploria clivosa & $\mathrm{x}$ & $\mathrm{x}$ & $\mathrm{x}$ \\
\hline Diploria labyrinthiformis & $\mathrm{x}$ & $\mathrm{x}$ & $\mathrm{x}$ \\
\hline Diploria strigosa & $\mathrm{x}$ & $\mathrm{x}$ & $\mathrm{x}$ \\
\hline Eusmilia fastigiata & $\mathrm{x}$ & $\mathrm{x}$ & $\mathrm{x}$ \\
\hline Favia fragum & $\mathrm{x}$ & $\mathrm{x}$ & \\
\hline Isophyllastrea rigida & $\mathrm{x}$ & $\mathrm{x}$ & $\mathrm{x}$ \\
\hline Isophyllia sinuosa & $\mathrm{x}$ & $\mathrm{x}$ & $\mathrm{x}$ \\
\hline Leptoseris cucullata & $\mathrm{x}$ & $\mathrm{x}$ & $\mathrm{x}$ \\
\hline Madracis decactis & $\mathrm{x}$ & $\mathrm{x}$ & $\mathrm{x}$ \\
\hline Madracis miriabilis & $\mathrm{x}$ & $\mathrm{x}$ & $\mathrm{x}$ \\
\hline Manicina aerolata & $\mathrm{x}$ & $\mathrm{x}$ & \\
\hline Meandrina meandrites & $\mathrm{x}$ & $\mathrm{x}$ & $\mathrm{x}$ \\
\hline Millepora alcicornis & $\mathrm{x}$ & $\mathrm{x}$ & $\mathrm{x}$ \\
\hline Millepora complanata & $\mathrm{x}$ & $\mathrm{x}$ & $\mathrm{x}$ \\
\hline Montastraea cavernosa & $\mathrm{x}$ & $\mathrm{x}$ & $\mathrm{x}$ \\
\hline Montastraea faveolata & $\mathrm{x}$ & $\mathrm{x}$ & $\mathrm{x}$ \\
\hline Montastraea franksi & & $\mathrm{x}$ & $\mathrm{x}$ \\
\hline Montrastraea annularis & & $\mathrm{x}$ & $\mathrm{x}$ \\
\hline Mussa angulosa & & $\mathrm{x}$ & $\mathrm{x}$ \\
\hline Mycetophyllia ferox & & $\mathrm{x}$ & $\mathrm{x}$ \\
\hline Mycetophyllia lamarckiana & $\mathrm{x}$ & $\mathrm{x}$ & $x$ \\
\hline Porites astreoides & $\mathrm{x}$ & $\mathrm{x}$ & $\mathrm{x}$ \\
\hline Porites divaricata & $\mathrm{x}$ & & $\mathrm{x}$ \\
\hline Porites furcata & $\mathrm{x}$ & $\mathrm{x}$ & $\mathrm{x}$ \\
\hline Scolemia cubensis & & $\mathrm{x}$ & $\mathrm{x}$ \\
\hline Siderastrea radians & $\mathrm{x}$ & $\mathrm{x}$ & $\mathrm{x}$ \\
\hline Siderastrea siderea & $\mathrm{x}$ & $\mathrm{x}$ & $\mathrm{x}$ \\
\hline Stephanocoenia intersepta & $\mathrm{x}$ & $\mathrm{x}$ & $\mathrm{x}$ \\
\hline Stylaster roseus & $\mathrm{x}$ & $\mathrm{x}$ & $\mathrm{x}$ \\
\hline
\end{tabular}



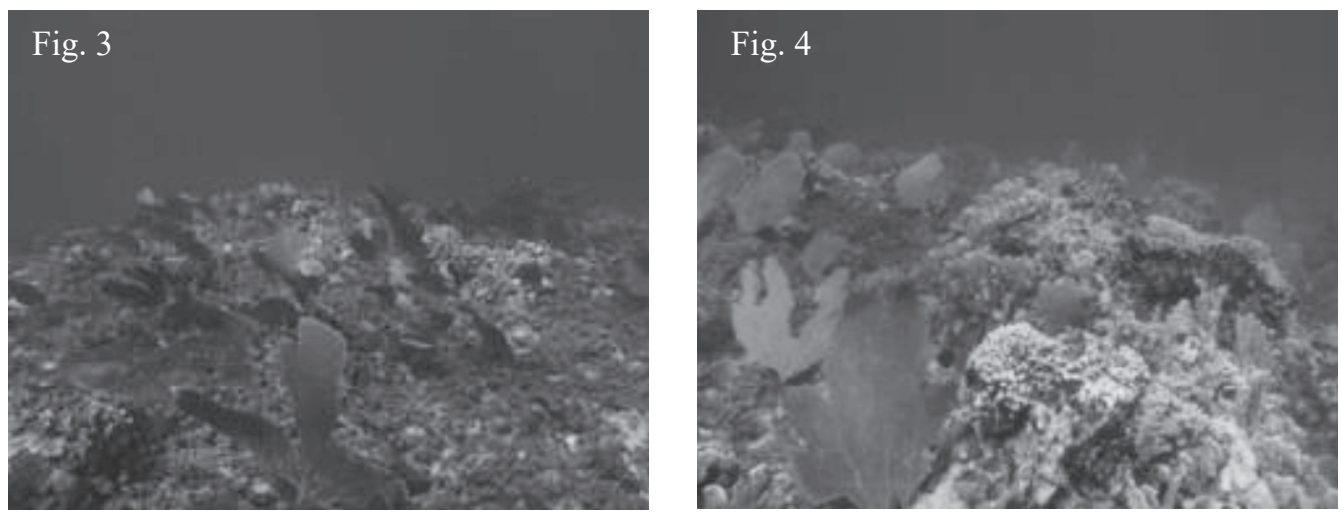

Figs. 3-4. Diferentes hábitats encontrados en el Banco de Serranilla durante el 2010; en las fotos 3 y 4 se observa el desarrollo de macroalgas, corales blandos (Gorgonia spp.), corales duros (Millepora spp. y Porites porites), entre 3 a $8 \mathrm{~m}$ de profundidad

Figs. 3-4. Different habitats found in the Serranilla Bank in 2010; figures $3 \& 4$ show the development of macroalgae, soft corals (Gorgonia spp.), hard corals (Millepora spp. and Porites porites); depth between 3 and $8 \mathrm{~m}$

representadas por Sargassum spp., Dictyota spp., Lobophora variegata, S. zonale y Halimeda spp. El desarrollo coralino se encuentra limitado a crecimiento en grietas o elevaciones de la terraza calcárea; la cobertura coralina fue de $15.7 \% \pm 7.9 \%$ y estuvo representada por Porites porites, P. astreoides, Siderastrea siderea, Stylaster roseus, Agaricia spp. y Montastraea cavernosa (Figs. 5-9). La riqueza de la comunidad coralina fue de 28 especies (Cuadro 1), que parece baja en comparación con las 45 especies más comunes encontradas en otras áreas del Archipiélago (Díaz et al. 1996a, b; 2000).

La categoría "esponjas" presentó el porcentaje más alto de cobertura de los tres sitios visitados durante la segunda expedición con $16.4 \% \pm 12.8 \%$, dominada por la especie Xestospongia muta (esponja barril), que en algunos de los puntos seleccionados alcanzó coberturas hasta del $40 \%$.

Debido a la profundidad de Bajo Alicia y días efectivos de campo por sitio, no fue posible la evaluación de la totalidad del complejo coralino.

\section{Bajo Nuevo}

La cobertura de coral encontrada en Bajo Nuevo fue de 30.2\% $\pm 19.1 \%$ (Fig. 2); el arrecife periférico de barlovento tiene un desarrollo similar a los demás atolones del Archipiélago. En la terraza prearrecifal se observó un buen desarrollo; hacia los extremos norte y sur de los dos sistemas arrecifales, en las zonas centrales existe la asociación Millepora-PalythoaPorolithon, como la descrita por Díaz et al. (1996a; 2000) para otras áreas del Archipiélago. La terraza lagunar es ancha, en algunos puntos es bastante somera (profundidades hasta de $4 \mathrm{~m}$ ) y se extiende a través de un plano en el cual se encuentran algunos setos y esqueletos de $A$. palmata, con costras de Diploria spp., P. astreoides y algas calcáreas. En las zonas más profundas de la laguna arrecifal abundan los parches coralinos. La laguna arrecifal tiene profundidades entre 6 y $20 \mathrm{~m}$; en esta franja se encontraron coberturas de coral vivo de hasta un 70\%. Es la zona mejor desarrollada de Bajo Nuevo y está dominada ampliamente por colonias de Montastraea 

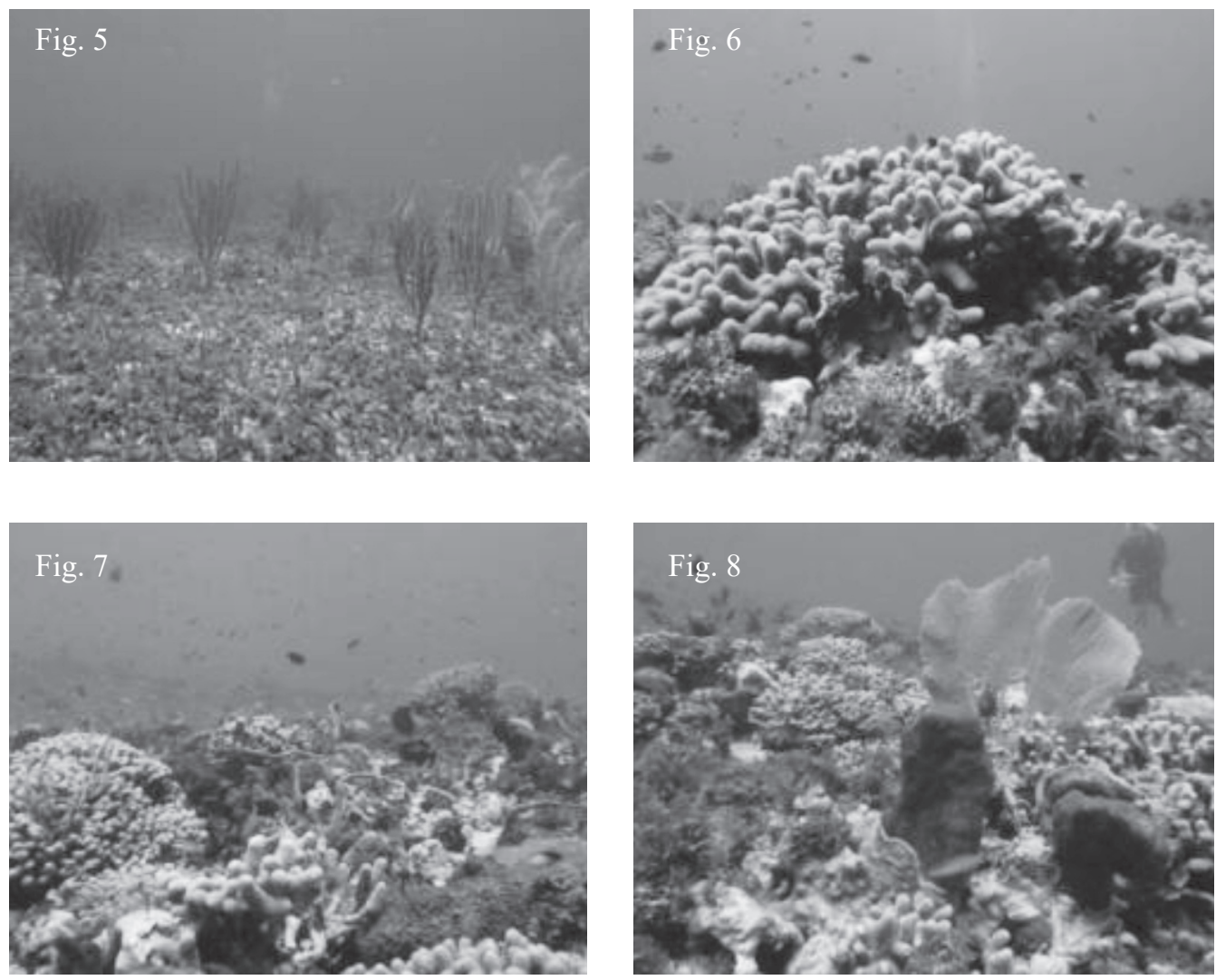

Figs. 5-8. Diferentes hábitats encontrados en el Bajo Alicia durante el 2010, en la figura 6 se observa el desarrollo de corales blandos (Pterogorgia spp.) dominando el sustrato y en las figuras 7-8 se observa el desarrollo de Porites porites, esponjas erectas, entre otros, dominando las elevaciones de roca coralina. Entre 15 a $20 \mathrm{~m}$ de profundidad

Figs. 5-8. Different habitats found in the Alice Shoal in 2010. Figure 6 shows the development of soft corals (Pterogorgia spp.) dominating the substrate and figures 7-8 show the development of Porites porites erect sponges among others, dominating coral rock elevations; depth between 15 and $20 \mathrm{~m}$

spp. Hacia la terraza arrecifal se registraron las mayores riquezas coralinas, observándose parches de Acropora cervicornis en buena condición (Figs. 9-12).

En la parte central de la laguna arrecifal, las profundidades son de aproximadamente 20 m o más; en esta fracción del complejo, el desarrollo coralino estuvo limitado a la elevación de estructuras calcáreas sobre un fondo de sedimento bioturbado. Allí la cobertura coralina disminuyó y la cobertura de los corales Montastraea spp. fue menos homogénea. La riqueza de la comunidad coralina en Bajo Nuevo fue de 37 especies de corales (Cuadro 1).

El componente de las algas tuvo una cobertura de $27.3 \% \pm 10.5 \%$. Su desarrollo estuvo más relacionado con la profundidad; en zonas someras dominaron los corales y a medida que la profundidad aumentó las algas tomaron más importancia. Las especies dominantes fueron: $L$. variegata, Halimeda spp., Dictyota spp., Sargassum hystrix, Sargassum spp. y Rhipocephalus spp. 

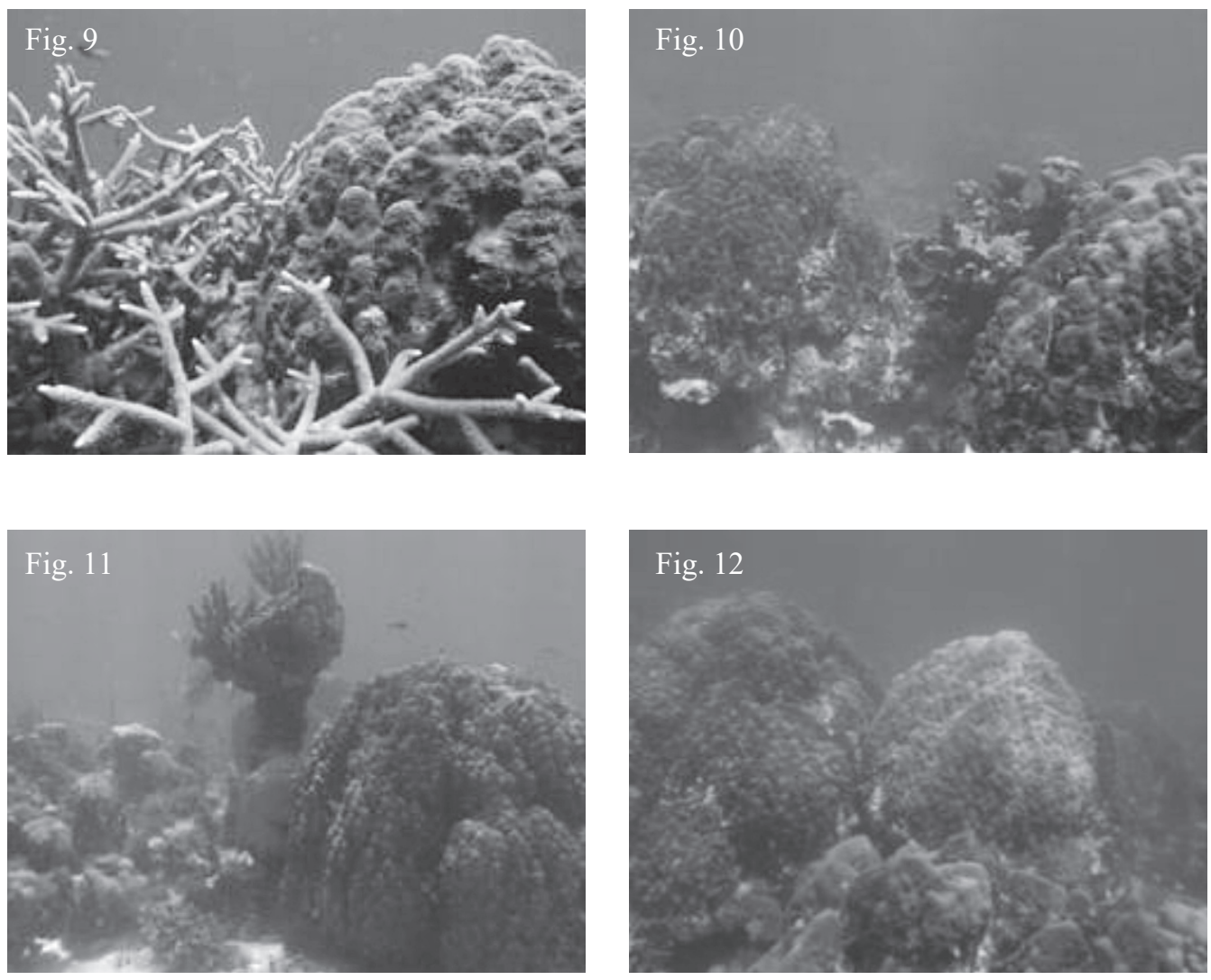

Figs. 9-12. Diferentes hábitats encontrados en Bajo Nuevo durante el 2010, se observa la dominancia de Montastraea spp. Entre 3-12 m de profundidad

Fig. 9-12. Different habitats found in New Shoal in 2010 showing dominance of Montastraea spp. Depth ranging from 3-12 m

\section{DISCUSIÓN}

Los arrecifes coralinos del mar Caribe han experimentado una notable pérdida de la cobertura coralina en las últimas décadas y en muchos casos el espacio ha sido ocupado por macroalgas frondosas, como consecuencia del enriquecimiento en nutrientes (Szmant, 2002; Gardner et al. 2003; Goreau, 2003). En el presente estudio se evidencia la proliferación de macroalgas en todos los bancos y bajos estudiados. Adicionalmente, se registra una alta densidad de octocorales y esponjas, que compiten por el espacio con los corales; las estructuras corresponden más a arrecifes de corales, esponjas y macroalgas, que en los últimos tiempos habrían sufrido un cambio de fase (phase shift) hacia un arrecife dominado por macroalgas (Lobophora, Dictyota, Turbinaria) acorde con lo planteado para diversas partes del mundo en Norström et al. (2009). Las algas han venido incrementándose en los últimos años en sus partes más someras y son el elemento dominante en muchos sitios, hasta unos $15 \mathrm{~m}$ de profundidad; luego tienden a disminuir, lo mismo que las esponjas, para dar lugar a arrecifes profundos $(>20 \mathrm{~m})$ con mayor 
dominancia de corales (Márquez et al. en prensa).

Los complejos arrecifales de Serrani1la, Bajo Alicia y Bajo Nuevo mostraron diferencias en el desarrollo y la complejidad de las estructuras coralinas, aunque se observa un patrón generalizado del estado del sistema. Las condiciones del arrecife periférico condicionan el desarrollo de los parches coralinos en la cuenca lagunar, y el nivel de energía del oleaje determina la composición biótica y la estructura de los arrecifes coralinos en función de la profundidad (Díaz, 2005). En el caso de Serranilla se observó una barrera arrecifal discontinua con un trazado rectilíneo, lo que permite el mayor ingreso de oleaje de mayor energía; este factor define la cobertura y el desarrollo de algunas especies de coral como Montastrea, que aunque fue observada, su desarrollo fue limitado a zonas profundas (Díaz, 2005), debido al requerimiento de baja energía. En el caso de Bajo Nuevo, la barrera permitió el desarrollo de parches coralinos masivos del género Montastraea, lo que refleja la predominancia de niveles de energía bajos; sobre la cuenca lagunar de las zonas convexas se observa el mejor desarrollo de este tipo de arrecifes, esta refracción de la barrera favorece también la extensión de áreas de setos de A. cervicornis. En el caso de Bajo Alicia se observó que las fuertes corrientes no permiten un desarrollo de grandes parches coralinos, solo se encuentran algunas colonias aisladas. La profundidad juega un importante papel en el desarrollo del complejo coralino. Pese a las anteriores diferencias en la morfología del arrecife periférico entre los atolones, la zonificación de los arrecifes está condicionada por la exposición y es muy similar a la descrita con anterioridad (Díaz et al. 1996a).
La cuenca lagunar es un área de depósito de sedimentos provenientes de la erosión de los arrecifes periféricos; el desarrollo de la laguna está altamente influenciado por la complejidad de la estructura, debido a la disipación de la energía del oleaje (Díaz et al. 1996a; 2000). En el caso de Bajo Nuevo se encontraron estructuras coralinas complejas que se desarrollan desde la terraza arrecifal hasta el talud del banco en la parte de sotavento. En la cuenca se desarrollan estructuras desde el fondo hasta la superficie, adicionalmente, los sedimentos depositados en la cuenca son bioturbados. Existe un gran número de parches coralinos en toda la cuenca lagunar que disminuyen al aumentar la profundidad. En el caso de Serranilla se encontró una amplia cuenca lagunar dominada por sedimentos gruesos y cascajo provenientes de fragmentos de coral que son arrastrados por la fuerte corriente y la acción del oleaje, esta falta de continuidad del arrecife periférico no favorece el desarrollo de ecosistemas de alta complejidad arrecifal.

En muchos ecosistemas arrecifales, la alta influencia de corriente y turbulencia no favorece el desarrollo de un mayor número de especies (Díaz et al. 1996a; García y Pizarro, 2002a), sin embargo, pese a las diferentes condiciones ambientales de los bancos, el número de especies encontradas en Bajo Nuevo y Bajo Alicia fueron similares, el desarrollo de las especies al parecer no está siendo influenciado por las condiciones ambientales, solo el desarrollo de los complejos arrecifales parece ser influenciado por estas condiciones.

En Bajo Alicia se encontró una terraza prearrecifal inclinada, que se extiende hasta unos $28 \mathrm{~m}$, posteriormente a esta profundidad aparece el talud que se desarrolló desde ese punto a profundidades abisales. Las fuertes condiciones ambientales no han 
permitido que exista una amplia complejidad; se encontró un desarrollo arrecifal tipo Porites en las márgenes más someras. Al igual que en todos los bancos y bajos del Archipiélago en profundidades por debajo de 18 m (Díaz et al. 1996b) se encontraron especies como C. natans, Montastraea spp. y D. cilindrus, asociadas a franjas y elevaciones de la terraza calcárea.

\section{CONCLUSIONES}

En general puede mencionarse que el desarrollo del ecosistema arrecifal es similar a las condiciones encontradas en otras zonas de la RB Seaflower y para el Caribe, donde en la mayoría de los casos las algas han tenido una fuerte dominancia en términos de cobertura. Como se observa en los casos de Serranilla y Bajo Nuevo, la presencia de un arrecife periférico y la profundidad juegan un importante papel en el desarrollo de la comunidad coralina. Es significativo mencionar que la cobertura de la categoría de corales duros en este sitio está entre una de las más altas del Archipiélago, posiblemente debido a la condición de aislamiento, lo que excluye la influencia del efecto de factores como la eutroficación y el impacto directo del hombre.

\section{AGRADECIMIENTOS}

Esta investigación fue posible gracias a la Corporación para el Desarro1lo Sostenible del Archipiélago de San Andrés, Providencia y Santa Catalina (CORALINA), la Gobernación Departamental del Archipiélago de San Andrés, Providencia y Santa Catalina y el Fondo de Compensación Ambiental. Gracias a todos los participantes.

\section{BIBLIOGRAFÍA}

Abril-Howard, A., Bolaños, N. \& Bent, H. (2010). Expedición científica de recolección de datos biológicos a Serrana, Roncador y primera aproximación al conocimiento de las comunidades coralina e íctica de los complejos arrecifales de Serranilla, Bajo Alicia y Bajo Nuevo-Colombia, sección norte de la Reserva de Biósfera Seaflower, Caribe Occidental 2010. San Andrés Isla, Colombia: CORALINA.

Acero-P., A. \& Garzón-Ferreria, J. (1994). Descripción de una nueva especie de Hyplopectrus (Pisces: Serranidae) del Caribe occidental y comentarios sobre las especies colombianas del género. An. Inst. Inv. Mar. Cost., 23, 5-14.

Barriga, E., Hernández, J. H., Jaramillo, I. J., Jaramillo, R., Mora, L. E., Pinto, P. \& Ruiz, P. M. (1969). La isla de San Andrés. Contribución al conocimiento de su ecología, flora, fauna y pesca. Bogotá D. C., Colombia: Instituto de Ciencias Naturales, Universidad Nacional. Dirección de Divulgación Cultural-Publicaciones.

Bolaños, N. (2006). Variaciones espaciales y temporales en la estructura de la comunidad de peces arrecifales de la Isla de San Andrés y su relación con el estado de los arrecifes. Tesis de pregrado no publicada, Universidad del Valle, Departamento de Biología, Cali, Colombia.

Bruckner, A. (2012). Global Reef Expedition: San Andres Archipelago, Colombia. Field Report. Maryland, EE.UU.: Khaled bin Sultan Living Oceans Foundation, Landover MD.

Chiquillo, M., Taylor, E., Howard, M. \& Mow, J. M. (2001). Coral Reef And Seagrass bed Condition, 1998-2000: San Andres CARICOMP Monitoring 
Site, Western Caribbean. San Andrés Isla, Colombia: CORALINA.

Dahlgren, C., Arboleda, E., Buch, K. L., Caldas, J. P., Posada, S. \& Prada, M. (2003). Characterization of reef-fish diversity, community structure, distribution and abundance on three Southwestern Caribbean atolls: Quitasueño, Serrana, and Roncador Banks (Seaflower Biosphere Reserve), Archipelago of San Andres and Old Providence, Colombia. San Andrés Isla, Colombia: The Ocean Conservancy \& CORALINA.

Díaz, J. M., Garzón-Ferreira, J. \& Zea, S. (1995). Los arrecifes coralinos de la isla de San Andrés, Colombia: estado actual y perspectivas para su conservación. Colección Jorge Álvarez Lleras. Vol. 7. Bogotá D. C., Colombia: Academia Colombiana de Ciencias Exactas, Físicas y Naturales.

Díaz, J. M., Díaz, G., Garzón-Ferreira, J., Geister, J., Sánchez, J. A. \& Zea, S. (1996a). Atlas de los complejos arrecifales coralinos del caribe colombiano: I Archipiélago de San Andrés y Providencia complejos arrecifales oceánicos. Santa Marta, Colombia: INVEMAR. Serie de Publicaciones Periódicas/INVEMAR No. 3.

Díaz, J. M., Sánchez, J. A., Zea, S. \& Garzón-Ferreira, J. (1996b). Morphology and marine habitats of two southwestern Caribean atolls: Albuquerque and Courntown. Atol. Res. Bull., 435, 1-33. Díaz, J. M., Barrios, L. M., Cendales, M. H., Garzón-Ferreira, J., Geister, J., López-Victoria, M., Ospina, G. H., ParraVelandia, F., Pinzón, J., Vargas-Ángel, B., Zapata, F. A. \& Zea, S. (2000). Áreas coralinas de Colombia. Santa Marta, Colombia: Serie de Publicaciones Periódicas/INVEMAR No. 5.
Díaz, M. J. M. (2005). Esquemas espaciales de zonación ecológica y morfología de las lagunas de los atolones y complejos arrecifales de un archipiélago oceánico del Caribe: San Andrés y Providencia (Colombia). Rev. Acad. Colomb. Cienc., 29(112), 357-369.

García, E. M. I. \& Pizarro, N. V. (2002a). Estado y biodiversidad de los arrecifes coralinos en las islas de Providencia y Santa Catalina. San Andrés Isla, Colombia: CORALINA.

García, E. M. I. \& Pizarro, N. V. (2002b). Estado y biodiversidad de los arrecifes coralinos en la isla de San Andrés. San Andrés Isla, Colombia: CORALINAFONADE. Ministerio del Medio Ambiente Colombia.

Gardner, T. A., Côté, I. M., Gill, J. A., Grant, A. \& Watkinson, A. R. (2003). Long-term region-wide declines in $\mathrm{Ca}$ ribbean coral. Science, 301, 958-960.

Garzón-Ferreira, J., Rodríguez-Ramírez, A., Bejarano, S., Navas-Camacho, R., Reyes-Nivia, M. C., Herrón, P., Zapata, F., Rojas, J. \& Caucalí, O. (2004). Estado de los arrecifes coralinos en Colombia en el año 2003. En INVEMAR (Ed.), Programa Biodiversidad de ecosistemas marinos, línea de monitoreo y conservación (pp. 79-135). Santa Marta, Colombia: INVEMAR, CORALINA, CEINER, Universidad del Valle.

Geister, J. (1973). Los arrecifes de la isla de San Andrés. Mitt. Inst. ColomboAlemán Invest. Cient., 7, 211-228.

Geister, J. (2001). Coral life and coral death in a recent Caribbean coral reef: a thirty-year record. Bull. Tohoku Univ. Mus., 1, 114-124.

Geister, J. \& Díaz, J. M. (1997). A field guide to the oceanic barrier reefs and atolls of the southwestern Caribbean (Archipelago of San Andres and 
Providencia, Colombia). Proc. 8th. Int. Coral Reef Symp., 1, 235-262.

Goreau, T. J. (2003, octubre). Waste nutrients: impacts coastal coral reefs and fisheries, and abatement via land recycling. Estudio de caso presentado en: United Nations expert meeting on waste management in small Island developing states. Havana, Cuba.

Herrón, P. (2004). Tendencias de cambio en la estructura y estado de los arrecifes coralinos de San Andrés, Providencia y Santa Catalina: Programa de Monitoreo de CORALINA durante el periodo 1998-2002. San Andrés Isla, Colombia: CORALINA-SIMAC.

Humann, P. \& Deloach, N. (2002a). Reef Coral Identification Florida, $\mathrm{Ca}$ ribbean, Bahamas. Jacksonville, Florida: New World Publications, Inc.

Humann, P. \& Deloach, N. (2002b). Reef Creature Identification Florida, Caribbean, Bahamas. Jacksonville, EE.UU.: New World Publications, Inc. INVEMAR. (2005). Capítulo II: Estado de los Ecosistemas Marinos y Costeros. 75-114. En INVEMAR (Ed.), Informe del estado de los ambientes marinos y costeros en Colombia. Santa Marta, Colombia: Serie de Publicaciones Periódicas/INVEMAR No. 8.

Márquez, G., Orozco, C. \& Pérez-García, M. E. (En prensa). Diversidad de corales en sitios de buceo en el Archipiélago de San Andrés y Providencia, Caribe occidental colombiano. Actualidades Biológicas.

Mow, J. M., Aguilera, C. \& Tabet, S. (2002). Archipiélago de San Andrés, Providencia y Santa Catalina: una reserva de Biosfera en el Caribe Colombiano. San Andrés Isla, Colombia: CORALINA.

Norström, A. V., Nyström, M., Lokrantz, J. \& Folke, C. (2009). Alternative states of coral reefs: beyond coral-macroalgal phase shifts. Mar. Ecol. Prog. Ser., 376, 295-306.

Pizarro, V. (2002a). Vulnerabilidady amenazas de los arrecifes coralinos de la Isla San Andrés. Proyecto: "Levantamiento de estudios y acciones para propiciar la recuperación y/o regeneración natural de los arrecifes coralinos en las aguas costeras de las Islas de San Andrés y Providencia”. Ministerio del Medio Ambiente Colombia. San Andrés Isla, Colombia: CORALINA-FONADE.

Pizarro, V. (2002b). Estado y biodiversidad de los arrecifes coralinos de la isla de San Andrés. Proyecto: "Levantamiento de estudios y acciones para propiciar la recuperación y/o regeneración natural de los arrecifes coralinos en las aguas costeras de las islas de San Andrés y Providencia”. Ministerio del Medio Ambiente. San Andrés Isla, Colombia: CORALINA-FONADE.

Pizarro, V. (2002c). Dinámica poblacional de corales (Scleractina: Faviidae) en la Isla de San Andrés, Caribe suroccidental: una aproximación. Tesis de posgrado no publicada, Universidad Nacional de Colombia. San Andrés Isla, Colombia.

Prada, M. (2005). Evaluation of marine monitoring implemented by coralina whitin the Seaflower Biosphere reserve advance report: coral reef monitoring. San Andrés Isla, Colombia: CORALINA.

Prahl, H. von \& Erhardt, H. (1985). Colombia, corales $y$ arrecifes coralinos. Bogotá D. C., Colombia: Fondo FEN-Colombia.

Rodríguez-Ramírez, A., Garzón-Ferreira, J., Bejarano-Chavarro, S., Navas-Camacho, R., Reyes-Nivia, C., Duque, G., Orozco, C., Zapata, F. \& Herrera, 
O. (2005). Estado de los arrecifes coralinos en Colombia. En INVEMAR (Ed.), Informe del estado de los ambientes marinos y costeros en Colombia: Año 2004 (pp. 77-114). Santa Marta, Colombia: Serie de Publicaciones Periódicas/INVEMAR No. 8.

Sánchez, J. A., Díaz, J. M. \& Zea, S. (1997). Gorgonian communities in two contrasting environments on oceanic atolls of the southwestern Caribbean. Bull. Mar. Sci., 61(2), 453-465.

Sánchez, J. A., Pizarro, V., Acosta, A. R., Castillo, P. A., Herrón, P., Martínez, J. C., Montoya, P. \& Orozco, C. (2005). Evaluating Coral Reef benthic Communities in remote Caribbean atolls (Quitasueño, Serrana, and Roncador banks, Colombia) multivariate approaches to recommend Marine Protected Areas for the Seaflower Biosphere Reserve (Archipelago of San
Andres and Providencia). Atoll. Res. Bull., 531, 1-65.

Sistema de Información Geográfica (SIGCORALINA). (2010). Centro de Análisis de Información Geográfica. San Andrés Isla, Colombia: CORALINA.

Szmant, A. (2002). Nutrient enrichment on coral reef: is it a major cause of coral reef decline? Estuaries, 25(4b), 743-766.

Weil, E. \& Ruiz, H. (2003). Guía para identificar los corales someros del Gran Caribe. Mayagüez, Puerto Rico: Departamento de Ciencias MarinasUniversidad de Puerto Rico.

Zea, S., Geister, J., Garzón-Ferreira, J. \& Díaz, J. M. (1998). Biotic changes in the reef complex of San Andres Island (Southwestwern Caribbean Sea, Colombia) occurring over nearly three decades. Atoll. Res. Bull., 456, 1-30. 